\title{
Las Naciones Unidas y los sistemas de alerta temprana: lecciones desde el escenario de África Occidental
}

\author{
United Nations and the early warning systems: \\ Lessons from the West African context
}

\author{
JARA CUADRADO \\ Instituto Universitario General Gutiérrez Mellado (UNED)

\section{Cómo citar/Citation} \\ Cuadrado, J. (2018). Las Naciones Unidas y los sistemas de alerta temprana: lecciones desde el escenario de África \\ Occidental. Revista Española de Ciencia Política, 46, 175-198. Doi: https://doi.org/10.21308/recp.46.08
}

\section{Resumen}

El contexto actual de seguridad internacional, caracterizado por un conjunto de riesgos globalizados, ha llevado a la Organización de las Naciones Unidas (ONU) a adaptar sus políticas para tratar de gestionar los complejos retos que se plantean. Ante este contexto, el presente artículo destaca el papel que tiene la prevención de conflictos, una tendencia que ha adquirido un mayor peso en los últimos años en la agenda de la comunidad internacional, así como el potencial de una de las herramientas que integra las políticas de prevención de conflictos: los sistemas de alerta temprana, cuya aplicación no se ha explorado lo suficiente. Con el fin de demostrar la importancia de este mecanismo en la gestión de los retos de seguridad a los que se enfrenta la comunidad internacional, se analiza la experiencia de la Comunidad Económica de los Estados de África Occidental (CEDEAO) en este campo, ya que su modelo de alerta temprana es citado como ejemplo desde la esfera internacional. Se plantean, además, las posibilidades que ofrece la cooperación en el terreno de la alerta temprana entre este organismo regional y las Naciones Unidas.

Palabras clave: prevención de conflictos, alerta temprana, regionalización de la seguridad, Naciones Unidas, CEDEAO.

\begin{abstract}
The current international security context is characterised by an array of globalised risks that has led the United Nations (UN) to adapt its policies to try to manage the complex challenges that are emerging. Within this context, the present article highlights the role played by conflict prevention - a trend that has gained more weight in the international community's agenda over
\end{abstract}


the last years, as well as the potential of one of the tools integrated into conflict prevention policies: the early warning systems, the application of which has not been sufficiently explored-. Aimed at demonstrating the importance of this instrument in managing the security challenges the international community faces, this article analyses the experience of the Economic Community of the West African States (ECOWAS) in this field, since its early warning model is used as an example within the international sphere. Besides, the article shows the possibilities the partnership ECOWAS-UN offers in the field of early warning.

Keywords: conflict prevention, early warning, regionalization of security, United Nations, ECOWAS.

\section{INTRODUCCIÓN}

Las transformaciones que han tenido lugar en el contexto internacional durante las dos últimas décadas y media han condicionado la agenda de paz y seguridad de Naciones Unidas (ONU), impulsando importantes cambios en sus políticas y operaciones de mantenimiento de la paz con el fin de adaptarse a las circunstancias cambiantes del escenario global.

La evolución en el terreno de la seguridad que se inició en los años noventa, tras el fin de la Guerra Fría (Hough, 2004), dio paso a un escenario donde empezaron a valorarse elementos que habían estado olvidados hasta entonces. Factores como la degradación medioambiental y las catástrofes naturales, el terrorismo, las crisis de refugiados, la violencia sexual como arma de guerra o las desigualdades sociales y económicas, se convierten en elementos fundamentales contemplados como amenazas para la paz y seguridad mundial.

El concepto de seguridad ha experimentado una evolución y ampliación, en convergencia con los desafíos de un mundo cada vez más globalizado, donde se produce una securitización de nuevos aspectos (Buzan et al., 1998). Los problemas, además, han adquirido un carácter transnacional, cuyos efectos atraviesan las fronteras. Se trata de factores de riesgo conectados, cuya relación se desarrolla en un escenario donde los cambios ocurren de forma rápida y las consecuencias se producen a escala mundial debido a las interconexiones que existen entre los actores globales. De esta manera, las actuales amenazas a la seguridad pueden tener efectos desestabilizadores más allá de su lugar de origen y acabar convirtiéndose en problemas regionales. Aspectos que antes se consideraban internos, se han convertido en factores de interés internacional.

Estos cambios conceptuales y normativos se traducen en nuevos retos para la agenda de la ONU. La organización ha tenido que impulsar y transformar nuevas líneas de actuación destinadas a la gestión de esas amenazas securitarias complejas. Dentro del abanico de medidas posibles, en este artículo se destaca el papel que tienen las políticas de prevención de conflictos, destinadas a evitar que una situación de tensión escale y derive en conflicto armado. En la actualidad, nos encontramos ante un 
posible cambio de paradigma en la doctrina de la prevención de conflictos, debido al interés creciente que esta recibe y el impulso que parece darse desde la llegada a la Secretaría General de la ONU de António Guterres, el 1 de enero de 2017.

La prevención de conflictos cuenta con diferentes mecanismos para controlar y gestionar el desarrollo de enfrentamientos armados y de otro tipo de situaciones violentas, incluyendo aquellas en las que se producen violaciones masivas de los derechos humanos. Uno de los instrumentos más interesantes es la alerta temprana. Se trata de una herramienta que sirve para identificar las amenazas existentes en un contexto de forma lo suficientemente efectiva como para que las medidas de prevención puedan ser llevadas a cabo. El papel que juegan los sistemas de alerta temprana en la prevención de conflictos armados y de otros retos importantes de seguridad se estudia en este artículo en el escenario de África Occidental, una región de importante interés estratégico para la comunidad internacional debido al conjunto de amenazas que alberga y la naturaleza transnacional de estas. Por otro lado, la elección de la zona responde al papel que la organización regional, la Comunidad Económica de los Estados de África Occidental (CEDEAO), juega tanto en materia de gestión de crisis como especialmente en el desarrollo de políticas preventivas aplicadas a contextos donde existen amenazas a la seguridad.

La CEDEAO es una organización regional con experiencia en operaciones de mantenimiento de la paz y un ejemplo en materia de prevención para el resto del continente africano. Sus misiones han sido repetidamente señaladas como exitosas y su política preventiva ofrece lecciones interesantes, por lo que se menciona como un modelo a seguir. Por esta razón, su trayectoria debe considerarse de gran interés en la agenda de prevención de la ONU y debe impulsarse una mayor colaboración entre ambas organizaciones.

El objetivo principal de este artículo es analizar el potencial y la aplicación de los sistemas de alerta temprana en contextos de interés para la seguridad internacional. Se pretende, por un lado, conocer la evolución de las políticas de prevención de conflictos y el papel de los mecanismos de alerta temprana y, por otro, analizar la experiencia y el potencial que tiene la CEDEAO en esta materia y las lecciones que pueden extraerse de su experiencia para la ONU. Teniendo en cuenta estos objetivos de investigación, la hipótesis que se plantea es que los sistemas de alerta temprana tienen un importante potencial en la gestión de crisis y que, sin embargo, no han recibido la suficiente atención en la agenda internacional. Para poder desarrollar este planteamiento se propone un estudio de caso a través de la experiencia de la CEDEAO en este campo.

La estructura del artículo se detalla a continuación. Primero, se presenta la doctrina de la prevención de conflictos y su evolución en la agenda internacional, para después centrar la atención en los sistemas de alerta temprana. Posteriormente, se hace hincapié en la importancia de la cooperación entre la ONU y las organizaciones regionales, teniendo en cuenta la tendencia actual hacia una mayor regionalización de la seguridad. Estos aspectos teóricos son analizados a través de un caso práctico, el de África Occidental y la CEDEAO. Una vez presentada la trayectoria de la organización africana en materia de prevención de conflictos, el texto analiza sus sistemas de alerta 
temprana con el fin de conocer el potencial que estos suponen para la gestión de futuros escenarios de conflicto en la zona. El artículo finaliza con una exposición de la ventana de oportunidades que ofrece el trabajo conjunto entre la ONU y la CEDEAO en esta materia.

\section{LA DOCTRINA DE LA PREVENCIÓN DE CONFLICTOS}

La celeridad y complejidad con la que aparecen y se expanden las actuales amenazas para la paz y seguridad sitúa a la ONU ante un reto permanente de reacción. En la trayectoria de las misiones de mantenimiento de la paz se han producido algunos puntos de inflexión importantes, en la mayoría de los casos como respuesta a fracasos a la hora de reaccionar a tiempo y de forma eficaz ante amenazas a la paz y seguridad internacional. El historial de la organización en la gestión de crisis pone en evidencia la falta de lectura de las causas estructurales de los conflictos o la ausencia de capacidad para adaptarse a los contextos en los que estos se desarrollan y actuar de forma temprana, como lo demuestran numerosos ejemplos ${ }^{1}$, predominando una cultura de reacción que la organización no ha logrado superar.

Desde mediados de los años noventa, la prevención de conflictos ha empezado a ocupar un espacio mayor en las líneas de actuación de la comunidad internacional, tanto en el seno de la ONU como en otras organizaciones regionales, como se pone de manifiesto en la mayor referencia a esta en la documentación oficial. Así, organizaciones regionales como la CEDEAO han convertido la prevención de conflictos en una de sus prioridades. Las catástrofes humanitarias que tuvieron lugar en esa década $^{2}$ y que significaron una mancha negra para la historia de la comunidad internacional, evidenciaron la importancia de la prevención, sobre todo si se tiene en cuenta que determinadas decisiones hubiesen podido evitarlas (Dorn y Matloff, 2000).

La ONU ha desarrollado diferentes medidas para la gestión de crisis. Estas pueden resumirse de la siguiente manera: prevención de conflictos; establecimiento de la paz; mantenimiento de la paz, y construcción de la paz (Naciones Unidas, 2008a: 17-18). Algunas de esas medidas se aplican en un momento determinado de la crisis y otras abarcan distintas fases, por lo que no pueden entenderse como medidas aisladas o conceptos estancos. Además, algunos de esos términos son muy amplios y abarcan aspectos que también están presentes en otras medidas, lo que lleva, en ocasiones, a confusiones entre los distintos términos. Pero esta divergencia no debe entenderse como una contradicción, ya que las distintas fases de una crisis se relacionan entre sí y, por lo tanto, las medidas destinadas a la gestión de la misma se complementan. La prevención de conflictos constituye un amplio abanico de tareas destinadas a evitar

1. Algunos casos recientes en el continente africano son: República Centroafricana, Sudán o Burundi.

2. Especialmente en Somalia, Bosnia y Ruanda. 
que se desencadene la violencia o que un conflicto activo empeore, por lo que se pone en marcha en las primeras fases de la gestión de crisis, pero debe estar presente a lo largo de toda la crisis.

La prevención de conflictos constituye uno de los rasgos distintivos y principal propósito de la ONU, tal y como se recoge en la Carta fundacional de la organización (Naciones Unidas, 1945). En este documento (capítulo VI) se exponen medidas preventivas como la mediación, la negociación o el arbitraje para el arreglo pacífico de controversias. Así nace el término de diplomacia preventiva, que empezó a ser utilizada a partir de la década de 1960 bajo la Secretaría de Dag Hammarskjöld. Posteriormente, en la Agenda para la Paz (1992), la prevención se diferenciaba de las acciones de peacemaking y peacekeeping, y en el Suplemento a la Agenda para la $P a z$ (1995) se contemplaba un punto de avance en el proceso de institucionalización de la prevención. La idea de diplomacia preventiva dará paso a otro tipo de medidas, que evolucionan a lo largo de las siguientes décadas, como se recoge en el informe del entonces secretario general Kofi Annan titulado Renovando las Naciones Unidas: un Programa de Reforma, donde se habla de despliegue preventivo ${ }^{3}$, desarme preventivo, acción humanitaria preventiva o desarrollo preventivo. Esta evolución se recoge en el gráfico 1.

Ya en el siglo XXI, las políticas de prevención de conflictos se convierten en un pilar fundamental para alcanzar la paz y seguridad internacional. En el Informe Brahimi (2001) se incidía en la importancia de la prevención, pues se consideraba «una opción menos costosa para la comunidad internacional que las acciones militares, el socorro humanitario de emergencia o la reconstrucción después de una guerra» (Naciones Unidas, 2000: 5-6). El mayor protagonismo que adquiere la prevención de conflictos en la agenda internacional a lo largo de la primera década del siglo xxi se refleja en varios documentos y medidas adoptadas ${ }^{4}$.

Un avance importante ha sido la constitución en 2014, del Equipo de Trabajo sobre Prevención de Conflictos (Inter-Departmental Task-Force on Conflict Prevention), que

3. Un ejemplo lo encontramos en el caso de Macedonia en 1992, donde se utilizó la fuerza como despliegue preventivo (Aguirre y Bruhn, 2002: 16-17).

4. Por ejemplo, el informe del secretario general titulado Prevención de Conflictos Armados de 2001, donde se hace una clasificación del tipo de medidas preventivas, la Resolución del Consejo de Seguridad 1366 de 30 de agosto de 2001 sobre el papel del Consejo en la prevención de conflictos armados, el Informe de la Asamblea general (A/RES/57/337) del 18 de julio de 2003 sobre prevención de conflictos armados, el informe del Grupo de Alto Nivel sobre las amenazas, los desafíos y el cambio del 2004, o el Informe del Secretario General sobre la aplicación de la resolución 1625 (2005) del Consejo de Seguridad relativa a la prevención de los conflictos, en particular en África, de 2008. Además, debido al interés que empezaba a despertar la doctrina de la prevención de conflictos en África, se crea en 2002 un Grupo de Trabajo Especial sobre la Prevención y la Solución de Conflictos en África ( $a d$ hoc Working Group on Conflict Prevention and Resolution in Africa), encargado de informar al Consejo de Seguridad de cualquier situación ante la que fuese necesario actuar. 
actúa «como mecanismo interinstitucional [y sirve] de foro para dar a conocer asuntos relativos a la prevención de conflictos, comunicar información y determinar las prioridades del sistema de las Naciones Unidas» (Naciones Unidas, 2015a: 13).

\section{Gráfico 1.}

EVOLUCión DEL CONCEPTO ‘ACCIÓN PREVENTIVA' EN LA ORGANIZACIÓN DE NACIONES UNIDAS

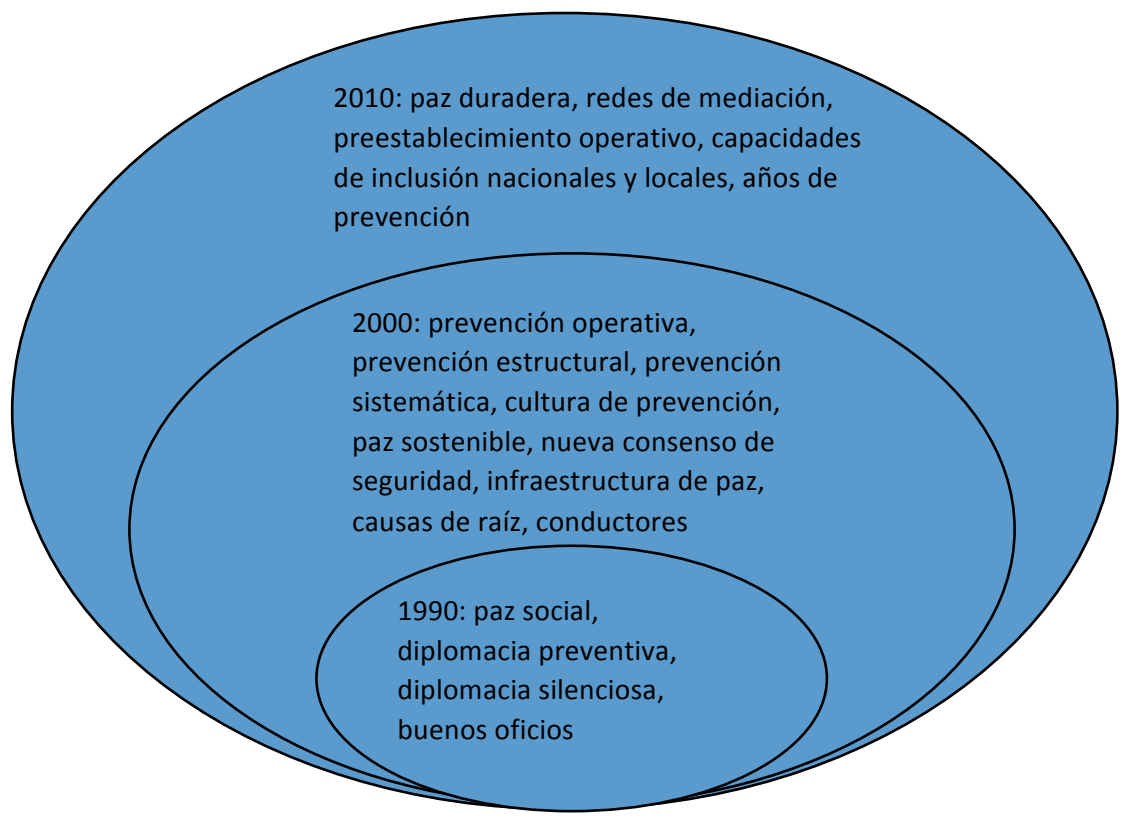

Fuente: elaboración propia adaptado de Muggah y White (2013: 3).

El Consejo de Seguridad tiene un papel fundamental en la prevención de conflictos que, sin embargo, no se ha reclamado con suficiente insistencia. Recientemente, el representante permanente de Reino Unido en la ONU recordaba la obligación de este órgano decisorio de garantizar que todos los Estados cumplan con su responsabilidad de prevenir conflictos, siguiendo el principio de responsabilidad de proteger (Rycroft, 2017) .

Tal y como reconocía el anterior secretario general, Ban Ki-moon, la experiencia ha demostrado que la inversión en prevención es menos costosa y que «dar prioridad

5. En este gráfico se podría añadir en un futuro la nueva fase en la que está entrando la doctrina de prevención de conflictos con la nueva Secretaría General.

6. Este principio, aprobado por la Asamblea General de la ONU e incorporado al Documento Final de la Cumbre Mundial 2005, hace referencia a tres áreas de responsabilidad: prevenir, reaccionar y reconstruir. 
a la prevención de conflictos es, con diferencia, nuestra opción más pragmática y económica» (Naciones Unidas, 2015a: 4). A través de la prevención se pueden abordar las causas estructurales de los conflictos de manera más eficiente y duradera que con otro tipo de medidas, pues el objetivo final de las acciones preventivas es trabajar sobre los contextos antes de que surjan tensiones, es decir, transformar "conflictos real o potencialmente violentos en procesos pacíficos de cambio político y social» (Ramsbotham et al., 2011: 113), situando a la prevención como instrumento de cambio social.

Con el despliegue de medidas preventivas se busca evitar que se desencadenen situaciones de violencia e inseguridad que pueden llevar a un conflicto armado y prevenir la escalada de violencia. Así, la ONU contempla la prevención de conflictos como aquellas acciones y medidas diplomáticas para gestionar las tensiones y evitar que se conviertan en conflictos violentos. La prevención de conflictos cuenta con diversas posibilidades: desde herramientas como la diplomacia preventiva, los buenos oficios y la mediación como instrumentos preventivos ante situaciones de guerra hasta otro tipo de acciones más completas, adaptadas a la complejidad de los contextos conflictuales. Debido a que el tipo de conflictos en los que se deben poner en marcha las medidas preventivas tiene diferentes elementos que se interrelacionan, para que la prevención sea efectiva «requiere de una estrategia integrada por diferentes sectores (diplomático, militar, político, económico, social y cultural) y con diferentes periodos de actuación» (García, 2002: 23). Son necesarias tanto la prevención estructural como la operativa, es decir, una combinación de medidas.

Siguiendo la clasificación que en su día estableció la Comisión Carnegie para la Prevención de Conflictos Mortales en su informe final titulado La Prevención de Confictos Letales (Preventing Deadly Conflict) de 1997, y que han adoptado otros organismos como la Comisión Europea, la ONU, la Unión Africana (UA) o la CEDEAO, se pueden diferenciar dos tipos de prevención:

- operativa, caracterizada por medidas que se ponen en marcha ante una crisis inminente. Son medidas que actúan cuando se presentan factores de riesgo y son directas, a corto plazo, como la mediación, la diplomacia preventiva, o las sanciones económicas.

- Estructural, con medidas que abordan las fuentes de los conflictos, las causas de fondo, e implican procesos de cambio a largo plazo. Son medidas para la estabilidad económica, social y política, como los programas de ayuda al desarrollo o las reformas en el sector de la gobernanza.

Una tercera categoría, entendida como la prevención sistemática ${ }^{7}$, aparece en el informe de 2005 Un Mundo Más Seguro: la responsabilidad que compartimos, del

7. Esta también aparece en otros documentos, como en el Informe del secretario general sobre la prevención de conflictos armados del 18 de julio de 2006 (A/60/891). 
Grupo de Alto Nivel sobre las amenazas, los desafíos y el cambio. Se centra en el tipo de amenazas transnacionales y se entiende como un tipo adicional de prevención de conflictos «que busca reducir los conflictos a nivel global con mecanismos que no estén enfocados en un Estado en particular» (Rubin y Jones, 2007: 393). Se trata de una forma comprehensiva de entender la prevención, que demuestra la evolución conceptual que ha experimentado la agenda de la ONU. En el documento citado sobre la aplicación de la Resolución 1625, por ejemplo, se pone de manifiesto cómo de la línea de diplomacia preventiva (los buenos oficios) se aspira a caminar hacia un enfoque más amplio, entendiendo la prevención de conflictos como

la prevención estructural para hacer frente a las causas profundas de los conflictos; la prevención operacional para asegurar el funcionamiento efectivo de los mecanismos de alerta temprana, mediación, acceso humanitario y respuesta, la protección de los civiles y las sanciones selectivas frente a las crisis inminentes; y la prevención sistémica para evitar que los conflictos existentes se extiendan a otros Estados (Naciones Unidas, 2008b: 3).

La evolución del tipo de medidas destinadas a la prevención muestra cómo esta doctrina ha ido evolucionando y adquiriendo un mayor protagonismo en el seno de la comunidad internacional.

António Guterres, el actual secretario general, parece que quiere focalizar más esfuerzos en la prevención, e incluso darle un papel protagonista, pues ha señalado que es necesario «un «nuevo enfoque integral» para salir de la tendencia actual en la que se gasta más tiempo y recursos en el conflicto que en prevenirlo» (Leone, 2017). La organización se encuentra actualmente en un proceso de revisión y de cambios conceptuales. En la primera intervención de Guterres como secretario general ante el Consejo de Seguridad, el 10 de enero de $2017^{8}$, la prevención ocupó un espacio importante de su discurso, señalando que la organización tiene que concentrar sus esfuerzos en «surge in diplomacy for peace» (Leone, 2017) es decir, en fomentar la diplomacia como medida para garantizar la paz, como herramienta preventiva. Se trata de una vuelta a los orígenes de la organización. Las bases en las que propone apoyarse el secretario general son: el trabajo conjunto con las organizaciones regionales; la movilización de actores influyentes, como los líderes religiosos y las organizaciones de la sociedad civil; un mayor desarrollo y apoyo a las capacidades de mediación de los órganos regionales y nacionales, así como utilizar con más frecuencia las opciones que recoge la Carta (en su capítulo VI) sobre la resolución pacífica de controversias (Mahmoud, 2017).

De esta manera, podrían darse pasos más definitivos hacia la consolidación de la prevención. Superar la cultura de reacción, que significa que las acciones se desarrollan una vez que la violencia ha estallado, ha sido uno de los objetivos prioritarios de la organización internacional desde que se creó. A pesar del mayor protagonismo que

8. Esta información se puede consultar en https://www.un.org/press/en/2017/sc12673.doc.htm. 
la prevención de conflictos ha adquirido en la agenda internacional, esta sigue siendo una tarea pendiente (Rubin, 2005; Muggah y White, 2013). Así lo demuestra el fracaso de las medidas desarrolladas por la ONU en crisis recientes como las que viven la República Centroafricana o Sudán del Sur (Global Centre for the Reponsibility to Protect, 2017; Burke y Pilkington, 2017).

La realidad muestra, por tanto, un avance importante en la documentación oficial y en las intenciones políticas que, sin embargo, no se ha traducido con éxito sobre el terreno.

\section{LOS SISTEMAS DE ALERTA TEMPRANA EN LA AGENDA DE LA ONU: UNA HERRAMIENTA PARA LA GESTIÓN DE LOS RETOS DE SEGURIDAD INTERNACIONALES}

Para poder superar la teoría y pasar a la acción, existen numerosas herramientas que trabajan de forma preventiva sobre los contextos y situaciones que amenazan la paz y la seguridad. De especial interés es el potencial de los sistemas de alerta temprana y su aplicación para la gestión de los retos de seguridad presentes en la agenda de la ONU. Se trata de un instrumento que ofrece importantes oportunidades para escenarios futuros de crisis. Con un mayor y mejor estudio de sus capacidades, se pueden elaborar recomendaciones para los políticos tanto locales como internacionales acerca de las opciones posibles orientadas a la acción preventiva.

Los sistemas de alerta temprana tienen la misión de identificar las amenazas existentes en un contexto de forma lo suficientemente efectiva y a tiempo como para que las medidas de prevención puedan ser llevadas a cabo. Tras definir unos factores (o variables) como indicadores de riesgo, esta herramienta monitoriza y recoge información en zonas de conflicto potencial previamente seleccionadas para controlar los cambios que pueden experimentar esas variables. La recogida de información se produce gracias a una red de actores que se encuentran sobre el terreno, como pueden ser los miembros de organizaciones no gubernamentales y los agentes humanitarios, y utiliza sistemas informatizados de datos. La información viaja del terreno a los centros de análisis ${ }^{9}$, donde los expertos deben verificar y evaluar los datos recibidos. Estos se transmiten a los actores y órganos pertinentes encargados de la toma de decisiones (decision makers) para que se desarrollen medidas tempranas, o lo que es lo mismo, acciones preventivas. El éxito de la alerta temprana se basa, por tanto, en que la recogida, análisis y comunicación de los datos sea a tiempo y se traduzca en la puesta en marcha de respuestas tempranas, cumpliendo así con el objetivo de la prevención. La tabla 1 muestra un resumen del funcionamiento de los sistemas de alerta temprana.

9. Las principales organizaciones internacionales y regionales, como la ONU, la Unión Europea, la UA o la CEDEAO, cuentan con oficinas conocidas como «situation rooms», a las que llegan datos relacionados con crisis internacionales y zonas de riesgo. 
TABLA 1.

FASES DEL FUNCIONAMIENTO DE LOS SISTEMAS DE ALERTA TEMPRANA PARA LA PREVENCIÓN DE CONFLICTOS ARMADOS

\begin{tabular}{ll}
\hline \multicolumn{1}{c}{ Fases } & \multicolumn{1}{c}{ Proceso } \\
\hline Observación y monitorización & $\begin{array}{l}\text { Selección de los indicadores o variables que van a ser } \\
\text { observadas (indicadores de riesgo) y sobre las cuales va a } \\
\text { realizarse un seguimiento con el objetivo de monitorizar } \\
\text { su evolución. }\end{array}$ \\
\hline Recogida de la información & $\begin{array}{l}\text { Herramientas y actores que trabajan en la recogida de } \\
\text { datos, ya sea sobre el terreno (field reporters) o desde el } \\
\text { exterior, a través de intercambios de información y con } \\
\text { sistemas informáticos programados. Papel clave de los } \\
\text { actores locales. }\end{array}$ \\
\hline Verificación y análisis & $\begin{array}{l}\text { Del terreno a los centros de análisis. Validación e } \\
\text { interpretación de la información primaria. Valoración } \\
\text { del riesgo y desarrollo de un posible escenario. }\end{array}$ \\
\hline \multirow{2}{*}{ Transmisión para generar } & $\begin{array}{l}\text { Llegada de los análisis a los actores y órganos } \\
\text { pertinentes encargados de la toma de decisiones } \\
\text { respuestas }\end{array}$ \\
(decision makers) para que se desarrollen medidas \\
tempranas (acciones preventivas).
\end{tabular}

Fuente: elaboración propia.

Los primeros estudios sobre alerta temprana se desarrollaron en los años cincuenta en el campo de la inteligencia y el reconocimiento militar. Una segunda fase de evolución tiene lugar en los años ochenta, cuando ese tipo de sistemas se amplían a estudios sobre la anticipación de desastres naturales o crisis humanitarias o a otros estudios de corte económico, como las condiciones del mercado. Esa perspectiva humanitaria de la alerta temprana la encabezaban organizaciones de asistencia no gubernamentales que trabajaban sobre crisis de refugiados, hambrunas o desastres ambientales, como por ejemplo Relief Web o la Oficina para la Coordinación de Asuntos Humanitarios (OCHA).

Fue en la década de los noventa cuando los sistemas de alerta temprana se empiezan a utilizar con el objetivo de prevenir la violencia y garantizar la paz y seguridad mundial. En ese contexto de posguerra fría se publica la ya mencionada Agenda por la paz del secretario general Boutros-Ghali, cuando la «cultura preventiva» empieza a ocupar más espacio en organismos internacionales y regionales.

Estos sistemas son utilizados hoy día en distintos contextos y campos, con el fin de detectar factores de riesgo y anticiparse a ellos. Por citar algunos ejemplos, se pueden utilizar para advertir sobre violaciones de derechos humanos, movimientos de armas pequeñas, tensiones intergrupales, emergencias humanitarias complejas (en las que intervienen diversos factores a la vez), cambios de tendencias en los mercados, procesos de radicalización entre determinados grupos sociales, etc. 
La alerta temprana enfocada en la prevención de conflictos armados y crisis humanitarias lleva años en la agenda de las organizaciones internacionales. Por ejemplo, en el Informe Brahimi se animaba a la ONU a convertirse en un actor más activo en la alerta temprana, con la capacidad de «proporcionar una ayuda más sólida a la comunidad, al país o a una región, para alertar de un conflicto y para terminar la violencia» (Mesa, 2008: 43).

Desde entonces se han desplegado misiones sobre el terreno a través de diferentes agencias y organismos como el Programa de Naciones Unidas para el Desarrollo (PNUD) y su Oficina para la Prevención de Crisis y Recuperación. El Departamento de Asuntos Políticos (DAP) es el encargado de gestionar los temas relacionados con la prevención de conflictos. En el 2003 el DAP y el PNUD establecen un programa conjunto sobre la prevención de conflictos en determinados países considerados vulnerables o en tensión (Programa Conjunto sobre el Fomento de las Capacidades Nacionales para la Prevención de Conflictos), mientras que el Banco Mundial crea la Unidad de Prevención de Conflictos y Reconstrucción. Por su parte OCHA ha asumido cada vez más tareas relacionadas con la alerta temprana.

Documentos más recientes, como el plan del anterior secretario general Ban Ki-moon conocido como «programa de acción quinquenal del secretario general The Future We Want, del 25 de enero de 2012, reflejan la importancia que se da a la alerta temprana. En él se expone la necesidad de priorizar la alerta y respuesta tempranas para prevenir conflictos, a través de: la relación, recogida e integración de información a lo largo del sistema internacional; el apoyo a las capacidades nacionales para facilitar el diálogo, y garantizar que los buenos oficios, la mediación, la respuesta a crisis y los servicios de peacebuilding que realiza la ONU sean fácil y rápidamente desplegables (Naciones Unidas, 2012: 6).

La alerta temprana aparece como el punto de partida para cualquier labor preventiva en el último informe del secretario general sobre prevención de conflictos (2015). Esta herramienta es, por tanto, un instrumento imprescindible en las líneas de actuación de la organización y tiene un potencial para distintas situaciones y contextos que no puede ignorarse. Por ejemplo, en el campo económico, sanitario, ambiental o humanitario, pero también en el campo militar y de la seguridad.

En suma, la utilización de esta herramienta en los contextos actuales de crisis puede ser muy eficaz, integrándose como parte esencial de las políticas preventivas, ya que es la llave para elaborar una serie de estrategias tempranas que permitan actuar antes de que la violencia estalle o escale hacia el conflicto armado. Ahora bien, para que este instrumento sea aprovechado al máximo por la organización internacional, el trabajo conjunto con los actores locales es clave, pues son estos los que se encuentran sobre el terreno y conocen el contexto mejor que nadie, por lo que son más capaces de desarrollar una tarea de alerta temprana. En este sentido, el punto de inflexión que podría estar viviendo la doctrina de la prevención de conflictos desde la llegada del nuevo secretario general hace hincapié en un aspecto que tiene una larga trayectoria en el campo de la paz y seguridad, pero que en los últimos ańos ha cobrado un mayor protagonismo: la regionalización de la seguridad. 


\section{REGIONALIZACIÓN DE LA SEGURIDAD: LA EXPERIENCIA EN EL CONTINENTE AFRICANO}

En las dos últimas décadas y media se ha producido una transición en las misiones internacionales de mantenimiento de la paz (peacekeeping), encargadas de garantizar la paz y seguridad en todo el mundo, que ha llevado a centrar el protagonismo en los actores regionales. Esta práctica ha tenido especial acogida en el continente africano, donde, desde la iniciativa en Darfur con la operación híbrida conjunta de la Unión Africana y las Naciones Unidas (UNAMID) en 2008, se ha producido una clara tendencia en virtud de la cual organizaciones como la UA o la CEDEAO se encargan de sus propios asuntos en materia de paz y seguridad.

La implicación de las organizaciones regionales en temas securitarios (Berman, 2002; Moller, 2009) tiene importantes ventajas, entre las que se encuentra un mejor conocimiento del contexto y, por lo tanto, de las raíces de los conflictos. Esto hace que en materia de prevención y alerta temprana los actores regionales y locales jueguen un papel protagonista, respaldados por la comunidad internacional. Además, se trata de actores que, al conocer mejor el terreno, entienden las situaciones desde otra perspectiva, pueden mover recursos con mayor facilidad y rapidez y tienen un interés directo en las repercusiones y en las soluciones. Por ello, la ONU «está cada vez más comprometida en compartir responsabilidades en peacekeeping, especialmente en situaciones de fragilidad estatal y violencia protagonizada por actores no estatales» (Wulf y Debiel, 2009: 6).

La ONU, consciente de que las organizaciones regionales se encuentran en una situación privilegiada para comprender las causas profundas de los conflictos armados, y siguiendo el capítulo VIII de la Carta, apoyan las acciones de los organismos regionales para actuar en pro de la defensa de la paz y seguridad internacional (Naciones Unidas, 2014: 2). La cooperación con dichos organismos es en la actualidad un aspecto prioritario para $\mathrm{ONU}$, como prueban numerosos documentos ${ }^{10}$.

La organización promueve las iniciativas de estos actores regionales para preparar el terreno a futuras operaciones internacionales de mantenimiento de la paz. Esto es algo que ocurre con frecuencia en el continente africano, y donde el caso de Somalia -African Union Mission in Somalia (AMISON) - sería un modelo para seguir.

El trabajo conjunto entre la ONU y la UA en el ámbito del mantenimiento de la paz se plasma en distintos aspectos; por ejemplo, en el apoyo de la primera a la segunda en «la elaboración de políticas, directrices y programas de capacitación, en particular en las esferas de la reforma del sector de la seguridad, la reconstrucción después de los conflictos, las mujeres, y la paz y la seguridad, la protección de los civiles, incluida la

10. Por ejemplo, los informes del secretario general Ban Ki-moon del 29 de diciembre de 2011 (S/2011/805) y del 1 de abril de 2015 (S/2015/229), conocido como Alianzas para la paz: hacia las asociaciones de colaboración en cuestiones relacionadas con el mantenimiento de la paz, o la Resolución del Consejo de Seguridad 21/67 del 28 de julio de 2014 (S/RES/2167). 
protección de los niños, la prevención de la violencia sexual y por razón de género en los conflictos armados, las situaciones posteriores a los conflictos y la respuesta en esos casos» (Naciones Unidas, 2014: p.3).

Esta cooperación de ambas organizaciones, donde la UA abrió paso a operaciones de la ONU, se ha materializado en distintas misiones: transición de la Misión Africana en Burundi (AMIB) a la Operación de las Naciones Unidas en Burundi (ONUB) y de la Misión de la Unión Africana en el Sudán (AMIS) a la Operación Híbrida de la Unión Africana y las Naciones Unidas en Darfur (UNAMID). Otro ejemplo interesante es el de la Misión Internacional de Apoyo a Malí con Liderazgo Africano (AFISMA), convertida posteriormente en la Misión Multidimensional Integrada de Estabilización de las Naciones Unidas en Malí (MINUSMA).

Los organismos regionales africanos, como la UA o la CEDEAO, asumen cada vez con más frecuencia la gestión de sus problemas de paz y seguridad ${ }^{11}$, a través del desarrollo de nuevas políticas y mecanismos, y es, desde la última década, una constante la cooperación en esta materia.

Resulta interesante destacar en este punto la labor que desarrolla la CEDEAO. La organización, a través de su Grupo de Monitorización de la Cesación del Fuego (ECOMOG), supuso «la primera iniciativa africana regional en mantenimiento de la paz, desplegada en Liberia, Sierra Leona y Guinea-Bissau» (Wulf y Debiel, 2009: 14). De acuerdo con un estudio del Parlamento Europeo (2008: 16) «la experiencia de la CEDEAO en el campo de la paz y seguridad ofrece mucho para que el resto de África pueda aprender». El modelo de la CEDEAO en la gestión de conflictos ha servido de referencia en algunos aspectos a la UA.

A pesar de que los fines para los que se crea la organización en 1975 son principalmente económicos, en el tratado fundacional se recogen principios -como por ejemplo, la protección de los derechos humanos- que marcarán las actuaciones de la organización en temas de paz y seguridad, los cuales irán adquiriendo más importancia con el tiempo.

Las actuaciones en materia de paz y seguridad se basan en el Tratado fundacional (modificado en 1993), y especialmente en el Protocolo sobre el Mecanismo para la Prevención, Gestión, Resolución de Conflictos, Mantenimiento de la Paz y Seguridad, acordado en la Cumbre de Lomé en 1999. En el artículo 25 de este Protocolo se recoge que el Consejo de Mediación y Seguridad puede actuar en situaciones de conflictos violentos, violaciones masivas de derechos humanos, ante situaciones en las que gobiernos democráticamente elegidos han sido derrocados, o puedan serlo, y ante amenazas humanitarias y sobre la estabilidad regional.

La CEDEAO es la más activa de todas las organizaciones subregionales del continente y tiene una larga trayectoria en el despliegue de operaciones de mantenimiento

11. El número de operaciones lanzadas por la UA ha aumentado significativamente en los últimos ańos, además de ser el continente africano uno de los principales contribuidores a las operaciones de mantenimiento de la paz de la ONU. 
de la paz. Tras las intervenciones en Liberia (ECOMOG Liberia), Sierra Leona (ECOMOG Sierra Leona) y Guinea Bissau (ECOMOG Guinea-Bissau), donde empezó a poner en práctica sus incipientes herramientas de mantenimiento de la paz, la CEDEAO ha desplegado operaciones de diferente naturaleza, como misiones electorales en países como Guinea-Bissau, Nigeria, Togo, Guinea, Costa de Marfil, Burkina Faso, Níger, Benín, Cabo Verde y Ghana y más recientemente en Gambia, o intervenciones militares en Liberia (ECOMIL), Costa de Marfil (ECOMICI) o Guinea-Bissau (ECOMIB).

El papel de la CEDEAO en este campo ha sido una referencia para la comunidad internacional, incluida la UA. Así lo expresaba en 2015 el entonces representante especial del presidente de la Comisión de la UA, Augustine Philip Mahiga, quien señalaba que tanto la ONU como la UA «deben seguir el ejemplo de la CEDEAO a la hora de desplegar una misión de imposición de la paz» (CEDEAO, 2015).

La tendencia actual es hacia una mayor implicación de la organización en la gestión de las amenazas que existen en la región a través de este tipo de operaciones, y en cooperación con la ONU, así como con Estados que bilateralmente intervengan en la zona, como ha sido el caso de Francia con las operaciones Serval ${ }^{12}$ y Barkhane ${ }^{13}$. Para cumplir con tal objetivo, la CEDEAO -como también ha hecho la UA- ha dado un valor especial a las políticas preventivas, tratando de impulsarlas en diferentes contextos y valiéndose, entre otras herramientas, de su sistema de alerta temprana, que cuenta con la colaboración de la sociedad civil. Su experiencia se puede considerar un modelo para la ONU y un impulso a la colaboración entre ambas organizaciones en materia de prevención de conflictos.

\section{LA CEDEAO COMO MODELO EN EL DESARROLLO DE POLÍTICAS DE PREVENCIÓN Y ALERTA TEMPRANA}

Siguiendo la práctica de la comunidad internacional, las organizaciones del continente africano, empezando por la propia UA, han dado un peso importante a las políticas de prevención de conflictos, superando así obstáculos relacionados con los principios de soberanía y no intervención. En este sentido, la Carta Constitutiva de la UA (2001, artículo 4) contempla que, basándose en una decisión de la Asamblea de Jefes de Estado y de Gobierno, se puede intervenir en los Estados miembros ante graves circunstancias, como crímenes de guerra, genocidio y crímenes contra la humanidad, y se otorga a la UA el derecho a intervenir en un Estado miembro si existen serias amenazas para la estabilidad de un Gobierno legítimo, con el fin de restaurar la paz y seguridad.

12. Se trata de la intervención militar que protagonizaron Francia y el gobierno de Malí en este país a partir de enero de 2013 como reacción a los avances de grupos yihadistas hacia el sur del país.

13. Esta sustituyó a la anterior. 
La Organización para la Unidad Africana (OUA), antecesora de la UA, ya integraba la prevención como una de sus líneas de actuación a través del Mecanismo para la Prevención, Gestión y Resolución de Conflictos (creado en 1993). Este mecanismo disponía de un Órgano Central cuya tarea era analizar la información que recibía desde el terreno con el objetivo de alertar sobre las causas de inestabilidad, violencia e inseguridad en el continente. El desarrollo de este organismo reflejaba «el deseo de la OUA en centrarse en la prevención de conflictos [...] -dejando las áreas más costosas y complejas a las operaciones de mantenimiento de la paz y reconstrucción posconflicto de las Naciones Unidas (Cilliers, 2005: 3)-.

Este interés lo hereda la UA, que, a través de la constitución de la Arquitectura de Paz y Seguridad Africana -African Peace and Security Architecture (APSA) ${ }^{14}-$, incorpora la prevención y alerta temprana como objetivos prioritarios (Engel y Gomes, 2009) para abordar los desafíos en materia de paz y seguridad. El órgano ejecutor de la APSA es el Consejo de Paz y Seguridad -Peace and Security Council (PSC)-, que tiene autoridad para desarrollar, entre otros fines, medidas destinadas a la alerta temprana y la diplomacia preventiva (Unión Africana, 2002). Dentro del Departamento de Paz y Seguridad de la UA se integra una División de Prevención de Conflictos y Alerta Temprana.

Uno de los pilares de APSA $^{15}$ es el Sistema Continental de Alerta Temprana $(\mathrm{CEWS})^{16}$, que aún se encuentra en un proceso de mejora de su funcionamiento. Se trata de un instrumento diseñado para recibir y gestionar la información que llega desde el terreno a su órgano central, la situation room, para, posteriormente, transmitirla a los actores encargados de tomar las decisiones, como son la Comisión de la UA y el Panel de Sabios.

Uno de los pilares de la política de prevención de conflictos de la UA es la armonización y coordinación con las Comunidades Económicas Regionales (REC por sus siglas en inglés). Así se recoge en distintos documentos, como en el Protocolo del PSC. Para ello, en enero de 2008, la UA y las REC firmaron un Memorándum de Entendimiento para la Cooperación en el Ârea de Paz y Seguridad.

Para cumplir con sus objetivos, el sistema continental necesita la colaboración de los Mecanismos Regionales de Alerta Temprana (Regional Early Warning Mechanisms), ya que estos trabajan sobre el terreno y deben remitir la información al órgano

14. Se trata del sistema africano para abordar los desafíos en materia de paz y seguridad, que se crea a partir de 2002.

15. Junto al PSC y el CEWS, se encuentran: el Panel de Sabios (Panel of the Wise), institución dedicada a la diplomacia preventiva y la resolución de conflictos.; la Fuerza Africana de Reserva (African Standby Force), una fuerza que puede ser utilizada, por ejemplo, para despliegue preventivo, y que cuenta con cinco brigadas (dependientes de cada zona regional); y el Fondo para la Paz (Peace Fund).

16. El CEWS se crea a través del artículo 12 del Protocolo del Consejo de Paz y Seguridad de la UA, donde se especifican sus funciones, destinadas a facilitar la anticipación y prevención de conflictos violentos. 
central del CEWS. Existen en África dos modelos destacados de sistemas de alerta y respuesta temprana: uno es el CEWARN, el mecanismo de la Autoridad Intergubernamental sobre el Desarrollo de África Oriental (Intergovernmental Authority on Development), la organización integrada por Yibuti, Eritrea, Etiopía, Kenia, Somalia, Sudán y Uganda; y otro es el ECOWARN (ECOWAS Warning and Response Network), el Sistema de Alerta Temprana de la CEDEAO, que lleva en marcha desde el año 2003. Este último representa un modelo para el resto del continente y para el sistema continental y, por ello, el artículo centra la atención en él.

La CEDEAO ha desarrollado una actividad importante en diplomacia preventiva y mediación en procesos electorales pacíficos. Su sistema de alerta temprana se encarga de monitorizar y analizar indicadores sobre violencia, buena gobernanza, paz y seguridad, violaciones de los derechos humanos, conflictos sociales, o tensiones étnicas (CEDEAO, 2008). Este sistema, que fue establecido en línea con las bases del artículo 58 del Tratado revisado de la CEDEAO y con el Protocolo sobre el Mecanismo para la Prevención, Gestión, Resolución de Conflictos, Mantenimiento de la Paz y Seguridad, ${ }^{17}$ cuenta con el Departamento de Alerta Temprana (antes conocido como Centro de Observación y Monitorización y Vigilancia) situado en Abuja, como órgano principal. El sistema dispone de cuatro oficinas distribuidas por la región (zonal bureaus) ${ }^{18}$, que transmiten información al centro situado en Nigeria (Terefe, 2010).

Para que la información llegue a esta oficina central, se necesita un trabajo de colaboración regional entre los distintos Estados miembros. Cada país cuenta con monitores de campo (dos por cada país, lo que hace un total de quince) y comités locales desde donde se envía la información a cada una de las cuatro oficinas zonales, encargadas de reunir y analizar los datos en una base diaria, para después enviar la información a la oficina central. La base de datos de la que se sirve ECOWARN trabaja con una serie de indicadores de diferentes campos (económicos, políticos, sociales y de seguridad). Posteriormente, esos datos, convertidos en informes, se transmiten a la Comisión de la CEDEAO, con el fin de que se identifiquen posibles crisis emergentes, de monitorizar crisis latentes y otras situaciones de interés.

Un aspecto sin duda positivo ha sido la decisión de la CEDEAO de integrar en el sistema de alerta temprana a las organizaciones de la sociedad civil, que proporcionan un apoyo insustituible. La organización cuenta con el trabajo de la West Africa Network for Peacebuilding (WANEP), que colaboró con la CEDEAO durante el proceso de puesta en marcha del Sistema del Observación y Monitorización de la organización subregional. WANEP utiliza un modelo de trabajo de alerta temprana que

17. En 2008 la organización desarrolló su marco de actuación en prevención de conflictos -ECOWAS Conflict Prevention Framework (ECPF)-, dando también una gran importancia a la alerta temprana.

18. Estas oficinas se encuentran en Banjul (encargada de recibir y analizar la información de Gambia, Cabo Verde, Guinea-Bissau y Senegal), Cotonú (para Benín, Nigeria y Togo), Ouagadougou (para Burkina Faso, Costa de Marfil, Malí y Níger), y Monrovia (para Liberia, Ghana, Guinea y Sierra Leona). 
actúa conjuntamente con ECOWARN. Entre ambas se elabora un trabajo importante y complementario, pues mientras WANEP cuenta con información de primera mano por el contacto directo sobre el terreno, la CEDEAO tiene los recursos y el mandato de los Estados para intervenir.

La aplicación práctica de esta herramienta de alerta temprana se ha puesto de manifiesto en diferentes contextos de inestabilidad en la región. Sirvan de ejemplo los contextos electorales, momentos de observación clave para la alerta temprana, debido a los niveles de violencia que muchas veces se asocian a ellos. Este fue el caso del proceso electoral que tuvo lugar en Gambia a finales de 2016 y donde la participación de la CEDEAO y sus políticas preventivas han sido aplaudidas por toda la comunidad internacional.

Tras la negativa del presidente Yahya Jammeh de aceptar su derrota en las elecciones de diciembre de 2016, se produce un clima de tensión en el país que lleva a la CEDEAO a involucrarse, a través de una intervención militar, conocida como "Restaurar la Democracia», con el objetivo de respaldar los resultados de las elecciones ${ }^{19}$.

El ambiente de crisis que existía en el país y que se había deteriorado a lo largo del 2016, anunciaba la posibilidad de una escalada hacia la violencia. Así lo ponían de manifiesto factores como, por ejemplo, un mayor número de detenciones arbitrarias de las personas críticas con el presidente o de desapariciones forzosas (Amnistía Internacional, 2016). Ante tal contexto de crisis, la CEDEAO inicia labores diplomáticas y de mediación con el presidente Yahya Jammeh para que aceptase el resultado de las elecciones (CEDEAO, 2017). Finalmente, la CEDEAO decide intervenir el 19 de enero de 2017, con el respaldo de la ONU, forzando en poco tiempo a Jammeh a aceptar su derrota y la posterior consolidación de Adama Barrow como el nuevo presidente del país.

La CEDEAO ha cumplido con éxito la intervención. Esto podría haberse debido a factores como la experiencia de entrenamiento conjunto que tienen las fuerzas de los diferentes Estados miembros, las lecciones aprendidas de casos pasados (Liberia, Costa de Marfil, Guinea-Bissau), la capacidad de autofinanciación por parte de la organización ${ }^{20}$, lo que le permite actuar de forma rápida, además del hecho de que la ECOMIG se ha creado para facilitar la salida pacífica, y no como una intervención de naturaleza militar (Nantulya, 2017). Gambia ha supuesto un ejemplo del éxito de las políticas de prevención de la CEDEAO y podría servir de paradigma para futuras crisis en la región. Se ha demostrado cómo los sistemas de alerta temprana de la CEDEAO son capaces de generar respuestas tempranas; este despliegue preventivo ha servido para evitar que un contexto postelectoral de tensión condujese hacia una escalada de la violencia.

19. Para ello se ha apoyado en la Carta de la UA sobre Democracia, Elecciones y Gobernanza, así como en el artículo 25 del Protocolo sobre Mecanismo para la Prevención, Gestión, Resolución de Conflictos, Mantenimiento de la Paz y Seguridad.

20. La misión de la CEDEAO en Gambia ha sido autofinanciada, como también ha ocurrido en el caso de Guinea-Bissau (De Coning, 2017). 
El ejemplo de Gambia contrasta con situaciones como la de Burundi, donde tanto las organizaciones de la región como el propio sistema continental, CEWS, han sido incapaces de actuar preventivamente. El caso de Sudán del Sur es también llamativo. Así lo recordaba el representante permanente de Reino Unido en la ONU, remarcando la necesidad en la organización de implementar las políticas de prevención y los mecanismos de alerta temprana: «El mensaje que se transmite desde el Consejo el Consejo de Seguridad [de la ONU] es el de la desunión y confusión. Tuvimos una oportunidad el pasado año para ejercer presión sobre las partes en Sudán del Sur, pero fallamos en el momento más importante. No podemos quejarnos ahora del trágico status quo de ese país si nosotros mismos no estamos preparados para actuar» (Rycroft, 2017). Frente a estos casos de incapacidad, la intervención en Gambia puede considerarse un ejemplo a seguir para el resto del continente, que lleva años estancado en la implementación de sus políticas preventivas y del CEWS.

En la crisis de Gambia se han puesto en marcha medidas preventivas, acudiendo en primer lugar a la mediación y, finalmente, haciendo uso del despliegue preventivo de fuerzas. Se trata de una medida disuasoria que se utiliza antes de que un conflicto estalle o antes una situación de escalada hacia la violencia.

Los sistemas de alerta temprana suponen una herramienta eficaz para la gestión de amenazas como las que están presentes en África Occidental (movimientos de radicalización, flujo de armas, tráfico de personas, violencia electoral, etc.). Una utilización adecuada de estos mecanismos, que trabajan detectando factores de riesgo, conduce a la elaboración de respuestas tempranas, objetivo prioritario de ONU desde su nacimiento, pero que, sin embargo, ha permanecido relegado al plano teórico. La experiencia positiva de la CEDEAO, reconocida por la propia organización internacional, debe servir de ejemplo para el giro que parece que podría darse en este campo con la llegada del nuevo secretario general.

\section{CONCLUSIONES: LECCIONES PARA UNA FUTURA COOPERACIÓN ONU-CEDEAO EN PREVENCIÓN DE CONFLICTOS}

El actual secretario general de la ONU ha remarcado la necesidad de potenciar la prevención de conflictos a través de la colaboración con las organizaciones regionales (Leone, 2017). La ONU es consciente del potencial que supone mejorar el trabajo conjunto con estas organizaciones para hacer frente a los problemas de paz y seguridad. Se trata de una meta presente desde la creación de la organización y que ha recibido cada vez mayor atención. La organización, «reafirma su intención de examinar otras medidas para propiciar una cooperación más estrecha y operacional entre las Naciones Unidas y las organizaciones regionales y subregionales en las esferas de la alerta temprana, la prevención de los conflictos, el mantenimiento de la paz y la consolidación de la paz» (Naciones Unidas, 2014: 5). Además, la ONU destaca la importancia de esta cooperación, especialmente con la Unión Africana, para el desarrollo de respuestas tempranas ante crisis emergentes en el continente, 
dando prioridad a la prevención de conflictos por encima de otras tareas (Naciones Unidas, 2008c).

Los beneficios de esta colaboración se materializan, por ejemplo, en la operacionalización de la Fuerza de Reserva de la CEDEAO, gracias al apoyo recibido. Además, el secretario general adjunto de Asuntos Políticos de la ONU, Jeffrey Feltman, ha seńalado recientemente que «trabajar con actores locales y con las organizaciones regionales puede hacer a la ONU más efectiva en la prevención de conflictos» (Shaban y Rahman, 2017).

Las lecciones que se pueden extraer de la experiencia de la CEDEAO en materia de prevención de conflictos y en la utilización de los sistemas de alerta temprana son importantes para el futuro de la agenda de ONU. Por ejemplo, para el desarrollo de las operaciones de mantenimiento de la paz ante diversas situaciones. La alerta temprana es una herramienta muy útil para la fase primera de la gestión de crisis que contempla la agenda internacional y se debe incorporar en la dinámica de las operaciones. Esta permite alertar a tiempo de situaciones de inestabilidad con la posibilidad de desarrollar medidas de acción preventiva, superando esa cultura de reacción que impera en la organización internacional.

La experiencia reciente en Gambia ha demostrado la eficacia del sistema de alerta temprana de la CEDEAO. Ha significado un éxito en materia de prevención, apoyado en aspectos como: el trabajo conjunto de actores políticos y actores locales de la sociedad civil; el uso de la vía diplomática como primera opción, o la colaboración entre los Estados miembros. Estos puntos fuertes de la organización africana la convierten en un referente para futuras crisis en las que sea necesario activar mecanismos de alerta temprana y políticas preventivas.

La agenda de la ONU contempla la alerta temprana como una parte esencial de la prevención de conflictos, tal y como recogen numerosos documentos oficiales. Sin embargo, su utilización se ha visto reducida. Principalmente se ha usado en el terreno de los desastres ambientales, dejando de lado otros contextos clave para la paz y seguridad que requieren medidas de acción inmediatas.

Si se tiene en cuenta el actual escenario de seguridad, con elevadas cifras de violencia, no se puede afirmar que el impulso de medidas preventivas por parte de ONU haya sido lo suficientemente efectivo, lo que demuestra que existe un dominio de la cultura de reacción frente a la cultura preventiva. La ONU se caracteriza por haber sido, hasta ahora, incapaz de actuar lo suficientemente temprano ante las crisis emergentes (Security Council Report, 2017).

Tal y como se señala en el informe del Grupo Independiente de Alto Nivel (HIPPO), del 17 de junio de 2015, «la Organización y sus Estados Miembros no han adoptado una cultura de prevención. Los Estados miembros no han invertido lo suficiente en la lucha contra las causas últimas del conflicto y, por lo general, las Naciones Unidas no han podido intervenir en fases suficientemente tempranas de las crisis surgidas» (Naciones Unidas, 2015b: 11).

Los esfuerzos han estado concentrados principalmente en otro tipo de actividades, pues si se compara, por ejemplo, la mediación y las operaciones de mantenimiento de 
la paz, que cuentan con una larga trayectoria, con la prevención se observa que hay menos solidez en sus políticas e incluso existe divergencia de perspectivas (Leijenaar y De Carvalho, 2017). Hay, además, «un desequilibrio entre la financiación destinada a las operaciones de apoyo a la paz y la destinada a la prevención» (De Carvalho, 2017: 8). También, las propias organizaciones regionales están más centradas en medidas reactivas y a corto plazo, lo cual se traduce en un déficit en el enfoque de la prevención estructural, que trabaja sobre las raíces de los conflictos (De Carvalho, 2017).

Existen aspectos importantes que es necesario mejorar para el funcionamiento de las políticas preventivas, tanto en el seno de la ONU como de las organizaciones regionales. Estos pasos hacia una mayor operacionalización y compromiso son necesarios si se quiere utilizar el potencial de los mecanismos de alerta temprana en las operaciones peacekeeping.

En cuanto al continente africano, es fundamental impulsar la coordinación y armonización entre los diferentes mecanismos de alerta temprana que existen, fomentar el intercambio de información para la redacción de informes de alerta y formular opciones políticas factibles. Este es un paso complicado, ya que, en general, los Gobiernos de los distintos países son reacios a compartir información sobre aspectos relacionados con su soberanía, y permitir la intervención de un 'cuerpo' o 'agente' intergubernamental. Los Estados no están dispuestos a apoyar las políticas preventivas cuando son las situaciones de sus países las que se convierten en objeto de la alerta temprana (Institute for Security Studies, 2017). Incluso, algunos Estados africanos consiguen presionar al PSC para evitar que esas situaciones se incluyan en la agenda de la organización, a pesar de que existan alertas generadas por el sistema central, el CEWS (Institute for Security Studies, 2017). Este debate está también presente en el Consejo de Seguridad de la ONU. Para Gueterres, la base para traducir la alerta temprana en respuesta temprana es el fomento de la confianza entre los Estados miembros de la organización y de sus relaciones con la ONU (Leone, 2017).

En este sentido, se considera que el trabajo conjunto entre la ONU y la CEDEAO es clave para superar el salto que existe entre la alerta y la respuesta temprana. Una mayor colaboración entre ambas organizaciones, que hoy día está representada a través de la Oficina de Naciones Unidas para África Occidental (UNOWA por sus siglas en inglés ${ }^{21}$-la primera oficina regional de la ONU que trabaja sobre prevención de conflictos-, y que se focalice en la mejora de los mecanismos de alerta temprana, se presenta como una oportunidad para alcanzar una política de prevención de conflictos exitosa.

La escasez de recursos humanos (falta de formación de expertos en análisis de alerta temprana) y materiales (Institute for Security Studies, 2016) es otro obstáculo importante para el éxito de los sistemas de alerta temprana de las organizaciones

21. Esta se ha unido recientemente a la Oficina del Enviado Especial para el Sahel, dando lugar así a la Oficina de Naciones Unidas para África Occidental y el Sahel (UNOWAS, por sus siglas en inglés). 
regionales, que la ONU tiene la capacidad de solventar. Con la formación de expertos desde la ONU y la aportación de recursos, los avances podrían ser muy importantes.

Además, es necesario que se resuelva el problema de la diversidad de metodologías utilizadas para el análisis de los indicadores de riesgo y de los contextos conflictuales, pues lleva en muchas ocasiones a la duplicación de esfuerzos en la investigación. El trabajo conjunto de la ONU y la CEDEAO sobre este asunto sería fundamental.

La experiencia de la CEDEAO en la gestión de las amenazas transnacionales ha mejorado en los últimos años, a pesar de los importantes esfuerzos que aún debe realizar y de los obstáculos internos que existen. Un trabajo coordinado del sistema de alerta temprana de esta organización y de la ONU presentaría una ventana de oportunidad para el futuro de las operaciones de mantenimiento de la paz y de otros retos de seguridad presentes en la agenda de la organización internacional. Este tipo de coordinación permitiría identificar contextos de riesgo en los que desarrollar acciones de forma preventiva y así ganar terreno a otro tipo de intervenciones más costosas, duraderas y, como la experiencia ha demostrado, menos exitosas.

\section{Referencias}

Aguirre, Mariano y Cecilia Bruhn. 2002. Guerra y olvido: la Unión Europea y la prevención de conflictos en África subsahariana. Barcelona: Intermon Oxfam.

Amnistía Internacional. 2016. Gambia: Death in detention of key political activist. Disponible en: https://www.amnesty.org/en/latest/news/2016/04/gambia-death-indetention-of-key-political-activist/.

Berman, Eric. 2002. "African regional organizations' peace operations: developments and challenges", African Security Review, 11 (4): 33-44. Disponible en: https://doi. org/10.1080/10246029.2002.9628143.

Burke, Jason y Ed Pilkington. 2016. UN under pressure over «failure to act» during South Sudan rampage. The Guardian. Disponible en web: https://www.theguardian.com/world/2016/aug/17/un-under-pressure-over-failure-to-act-duringsouth-sudan-rampage.

Buzan, Barry, Ole Waever y Jaap Ole. 1998. Security: A New Framework for Analysis. Boulder: Lynne Rienner.

CEDEAO. 2008. Marco para la Prevención de Conflictos dentro de la CEDEAO, Regulación MSC/REG.1/01/08.

CEDEAO. 2015. ECOWAS gets AU's commendation on peace-keeping operations in Africa, ECOWAS Info. Disponible en: http://www.ecowas.int/ecowas-gets-auscommendation-on-peace-keeping-operations-in-africa/.

CEDEAO. 2017. ECOWAS Authority Chair, President Sirleaf Comments on ECOWAS Mediation Intervention in the Gambia. ECOWAS Info. Disponible en: http://www.ecowas.int/ecowas-authority-chair-president-sirleaf-comments-onecowas-mediation-intervention-in-the-gambia/. 
Cilliers, Jakkie. 2005. Towards a Continental Early Warning System for Africa. ISS Paper 102. Disponible en: https:/www.files.ethz.ch/isn/99193/PAPER102.pdf.

De Carvalho, Gustavo. 2017. Conflict prevention. What's in it for the AU? Institute for Security Studies, Policy Brief, 103. Disponible en: https://issafrica.s3.amazonaws. com/site/uploads/policybrief103-2.pdf.

De Coning, Cedric. 2017. Can the AU Finance its own peace operations, and if so, what would the impact be? Complexity 4 Peace Operations. Disponible en: https:// cedricdeconing.net/2017/03/28/can-the-au-finance-its-own-peace-operations-and-ifso-what-would-the-impact-be/.

Dorn, A. Walter y Jonathan Matloff. 2000. «Preventing the Bloodbath: Could the UN have Predicted and Prevented the Rwandan Genocide?», Journal of Conflict Studies, 20 (1). Disponible en: https://journals.lib.unb.ca/index.php/jcs/article/ view/4333/4968.

Engel, Ulf y João Gomes. 2009. "The African Union's New Peace and Security Architecture: Towards an evolving security regime?», African Security, 2 (2-3): 82-96. Disponible en: https://doi.org/10.1080/19362200903359774.

García, Bernardo. (2002). Una nueva oportunidad para la prevención de conflictos: análisis comparativo de las politicas de Estados Unidos y de la Unión Europea sobre la prevención de conflictos violentos. Bilbao: Universidad de Deusto.

Global Centre for the Responsibility to Protect. 2017. Population at Risk, Central African Republic. Disponible en: http://www.globalr2p.org/regions/central_african_ republic.

Hough, Peter. 2004. Understanding Global Security. Londres: Routledge.

Institute for Security Studies. 2016. PSC Interview: Disputed elections top the list of the AU's early warning for 2016. Peace and Security Council Report. Disponible en: https://issafrica.org/pscreport/addis-insights/psc-interview-disputed-elections-topthe-list-of-the-aus-early-warning-for-2016.

Institute for Security Studies. 2017. Denialism plagues Africa's early warning system. Peace and Security Council Report. Disponible en: https://issafrica.org/pscreport/ on-the-agenda/denialism-plagues-africas-early-warning-system.

Leijenaar, Annette y Gustavo De Carvalho. 2017. «We can honour UN peacekeepers by preventing conflicts», ISS Today. Disponible en: https://issafrica.org/iss-today/ we-can-honour-un-peacekeepers-by-preventing-conflicts.

Leone, Faye. 2017. "Conflict Prevention is 'The Priority' says UN Secretary-General», SDG Knowledge Hub. Disponible en: http://sdg.iisd.org/news/conflict-preventionis-the-priority-says-un-secretary-general/.

Mahmoud, Youssef. 2017. What Would It Take to Make a "Surge in Diplomacy for Peace» Work? International Peace Institute, Global Observatory. Disponible en: https://theglobalobservatory.org/2017/01/sustaining-peace-diplomacy-antonioguterres/.

Mesa, Manuela. 2008. La prevención de conflictos y la construcción de la paz en el seno de Naciones Unidas: de las palabras a la acción. CEIPAZ. Disponible en: http:// www.ceipaz.org/images/contenido/02.\%20manuela\%20mesa.pdf. 
Moller, Bjórn. 2009. The African Union as Security Actor: African Solutions to African Problems. Crisis State Research Centre, Working Paper, 59. Disponible en: http:// eprints.lse.ac.uk/28485/1/WP57.2Moller.AU.pdf.

Muggah, Robert y Natasha White. 2013. Is there a preventive action renaissance? The policy and practice of preventive diplomacy and conflict prevention? NOREF. Disponible en: http://noref.no/var/ezflow_site/storage/original/application/00175abccdb1df5f006c8e40da206643.pdf.

Naciones Unidas. 1945. Carta de las Naciones Unidas, capítulo VI: «Arreglo pacífico de controversias». Disponible en: http://www.un.org/es/sections/un-charter/chapter-vi/index.html.

Naciones Unidas. 2000. Informe del Grupo sobre las Operaciones de Paz de las Naciones Unidas (Informe Brahimi), A/55/305-S/2000/809 (21-7-2000).

Naciones Unidas. 2008a. United Nations Peacekeeping Operations. Principles and Guidelines. Nueva York: Secretariado de Naciones Unidas.

Naciones Unidas. 2008b. Informe del secretario general sobre la aplicación de la Resolución 1625/2005 del Consejo de Seguridad relativa a la prevención de conflictos, en particular en África. Consejo de Seguridad S/2008/18 (14-1-2008).

Naciones Unidas. 2008c. Security Council Expresses Determination to Strengthen Peace and Security Partnership With African Union, Following Day-Long High-Level Debate, Informe de prensa del Consejo de Seguridad, SC/9301, 16-4-2008.

Naciones Unidas. 2012. Programa de acción quinquenal del secretario general: «El futuro que queremos» (25-1-2012).

Naciones Unidas. 2014. Resolución 2167 del Consejo de Seguridad S/RES/2167 (28 de julio de 2014).

Naciones Unidas. 2015a. Informe sobre las Naciones Unidas y la prevención de conflictos: renovación del compromiso colectivo. Resolución del Consejo de Seguridad S/2015/730 (25-9-2015).

Naciones Unidas. 2015b. Informe del Grupo Independiente de Alto Nivel sobre las Operaciones de Paz: aunar nuestras ventajas en pro de la paz-politica, alianzas y personas. Asamblea General-Consejo de Seguridad A/70/95 (17-6-2015).

Nantulya, Paul. 2017. Lessons from Gambia on Effective Regional Security Cooperation. African Center for Strategic Studies. Disponible en: http://africacenter.org/spotlight/gambia-regional-security-cooperation/.

Parlamento Europeo. 2008. Options for the EU to Support the African Peace and Security Architecture. Disponible en: http://www.chathamhouse.org.uk/files/11637 0508eu_africa.pdf.

Ramsbotham, Oliver, Tom Woodhouse y Hugh Miall. 2011. Resolución de conflictos la prevención, gestión y transformación de conflictos letales. Barcelona: Bellaterra.

Rubin, Barnett R. y Bruce D. Jones. 2007. «Prevention of Violent Conflict: Tasks and Challenges for the United Nations", Global Governance 13: 391-408.

Rycroft, Matthew. 2017. Peacekeeping should exist alongside both conflict prevention and peace-building work. Foreign and Commonwealth Office. Disponible en: 
https:/www.gov.uk/government/speeches/peacekeeping-should-exist-alongside-bothconflict-prevention-and-peace-building-work.

Shaban, Alfa y Abdur Rahman. 2017. "UN lauds West Africa for successes in 'preventive diplomacy", Africanews. Disponible en: http://www.africanews. com/2017/03/07/un-lauds-west-africa-for-successes-in-preventive-diplomacy/.

Security Council. 2017. Can the Security Council Prevent Conflict? Security Council Report. Research Report, 1. Disponible en: http://www.peacewomen.org/sites/ default/files/research_report_conflict_prevention_2017.pdf.

Terefe, Birikit. 2010. Establishing an Early Warning System in the African Peace and Security Architecture: Challenges and Prospects. KAIPTC, Occassional Paper, 29. Disponible en: http://www.kaiptc.org/publications/occasional-papers/documents/ occasional-paper-29-birikit.aspx.

Unión Africana. 2002. Protocolo relativo al establecimiento del Consejo de Paz y Seguridad de la Unión Africana. Durban (South Africa): African Union.

Wulf, Hebert y Debiel, Tobias. 2009. Conflict early Warning and Response Mechanisms: Tools for Enhancing the Effectiveness of Regional Organizations? A Comparative Study of the AU, ECOWAS, IGAD, ASEAN/ARF and PIF. Crisis States Research Centre, Crisis States Working Paper Series, 2. Disponible en: http:// eprints.lse.ac.uk/28495/1/WP49.2.pdf.

Presentado para evaluación: 3 de julio de 2017.

Aceptado para publicación: 10 de diciembre de 2017.

\section{JARA CUADRADO BOLAÑOS}

jaracb.4488@gmail.com

Investigadora predoctoral en el Instituto Universitario General Gutiérrez Mellado (UNED). Estudiante de doctorado en Seguridad Internacional (UNED). Sus líneas de investigación se centran en los sistemas de alerta temprana para la prevención de conflictos armados, retos de seguridad en África, tráfico de personas, procesos de transición y estudios de paz. Ha realizado estancias de investigación en el Instituto Português de Relaçôes Internacionais (IPRI, Universidad Nova de Lisboa) y en la School of Oriental and African Studies (SOAS, Universidad de Londres), y trabajo de campo en Ghana. Cuenta con varias publicaciones nacionales e internacionales y en 2015 recibió el Premio de Ensayo Casa África. 